\title{
Group Synchronization Control Considering Difference of Conversation Roles
}

\author{
Kazuki Hosoya Yutaka Ishibashi Shinji Sugawara \\ Department of Scientific and Engineering Simulation \\ Nagoya Institute of Technology \\ Nagoya 466-8555, Japan \\ Email: k_hosoya@mcl.nitech.ac.jpｉshibasi@nitech.ac.jp
}

\author{
Kostas E. Psannis \\ Department of Technology Management \\ University of Macedonia \\ Thessaloniki 540 06, Greece \\ Email: kpsannis@uom.gr
}

\begin{abstract}
This paper proposes group (or inter-destination) synchronization control considering difference of conversation roles in multipoint communications where users have a conversation with each other by using voice and video. By subjective assessment, the paper also investigates the effects of the proposed control for networked rock-paper-scissors. Assessment results show that the control can maintain the comprehensive quality higher than the conventional control.
\end{abstract}

\section{INTRODUCTION}

In multipoint communications with voice and video, users can perform various kinds of work such as conversations and networked games [1]-[4]. However, temporal relationships of the media streams may be disturbed owing to network latency and jitter. These are main factors which damage the output quality of the media streams. The Quality of Experience (QoE) may be damaged if the output timings among multiple terminals are different from each other [5]. In [5], the authors investigate the influences of the network latency on the perception of fairness in a name-guessing task like fastest fingers first as networked competitive work using voice and video by subjective assessment. They show that the fairness among users is damaged by the difference in output timing among the users.

To solve such a problem, we need to carry out group (or inter-destination) synchronization control [6]-[10], which adjusts the output timing of media streams among multiple terminals. In [8]-[10], the authors demonstrate the effects of group synchronization control by objective assessment when a single media source multicasts voice and video to two media destinations. However, they handle only one-way transmission from the media source to the media destinations. In [11], where the authors deal with a networked shooting game in which two players fight with each other and the players transmit information about shots and movements of fighters through a server, the effect of group synchronization control in duplex transmission is illustrated by objective assessment. However, they do not examine how the group synchronization control affects the QoE since subjective assessment has not been carried out.

In [12], we subjectively assess the effects of the group synchronization control on fairness, group synchronization quality, interactivity, and comprehensive quality by dealing with a name-guessing task like fastest fingers first employed in [5] as competitive work and networked rock-paper-scissors (i.e., rock-paper-scissors over a network) as collaborative work. As a result, we show that the group synchronization control improves the fairness in name-guessing task like fastest fingers first. Also, we reveal that in networked rock-paperscissors, the effects of the group synchronization control are influenced by the difference of roles in conversation.

This paper proposes group synchronization control considering difference of conversation roles for networked rock-paper-

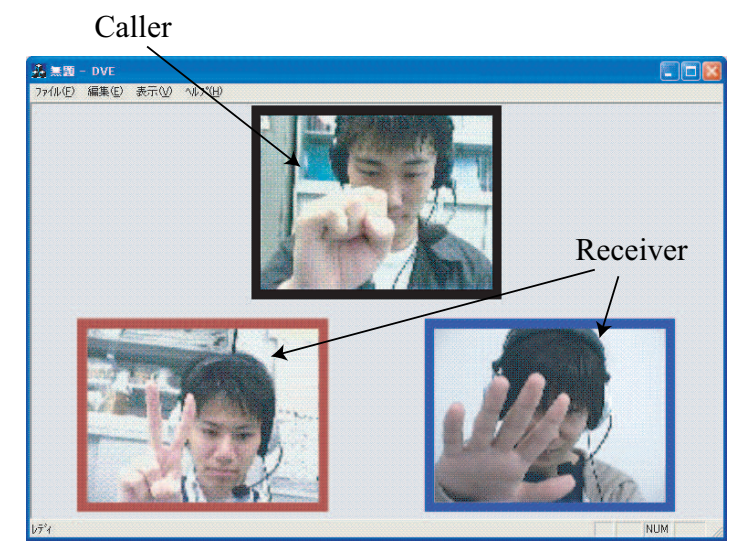

Fig. 1. Displayed images in networked rock-paper-scissors.

scissors handled in [12]. We also investigate the effects of the control by subjective assessment.

The rest of this paper is organized as follows. Section II describes networked rock-paper-scissors, and Section III explains the conventional group synchronization control. We propose the group synchronization control considering difference of conversation roles in Section IV. Then, our experimental system and the subjective assessment method are explained in Section V. Assessment results are presented in Section VI. Section VII concludes the paper.

\section{NETWORKED ROCK-PAPER-SCISSORS}

In networked rock-paper-scissors, there are one caller and two receivers, who collaborate with each other. The caller plays a role to say "Rock, paper, scissors, go!" The caller and the receivers try to show rock, paper or scissors at the same time (see Fig. 1).

\section{CONVENTIONAL GROUP SYNCHRONIZATION CONTROL}

For group synchronization control, we adopt the distributed control scheme [10] as in [12]. In the distributed control scheme, each terminal transmits information about the output timing of media units $(M U s)$, each of which is the information unit for media synchronization, at the terminal to all the other terminals. Thus, each terminal can know the output timings of all the other terminals.

The output timing of an MU is set to the generation time of the MU plus $\Delta(\geq 0) \mathrm{ms}$. The value of $\Delta$ is dynamically changed between $\Delta_{\mathrm{L}}(\geq 0) \mathrm{ms}$ and $\Delta_{\mathrm{H}}\left(\geq \Delta_{\mathrm{L}}\right)$ ms according to the network latency [13].

Each terminal determines the reference output timing [10], which is an output timing to which all the terminals try to 
adjust their output timings. We choose the latest output timing from among all the output timings as the reference output timing [10]. Each terminal achieves group synchronization by making its output timing approach the reference output timing [10]. Each MU generated at the terminal is also output $\Delta$ ms late at the terminal. The reason is that the output timing of the MU is adjusted to the output timings of MUs generated at the other terminals.

In networked rock-paper-scissors, there are two types of roles (i.e., caller and receiver). The caller sees each receiver show rock, paper or scissors the same time interval as the round-trip delay (i.e., about $2 \Delta \mathrm{ms}$ ) between the caller's and receiver's terminals after the caller has shown rock, paper or scissors at the caller's terminal. However, since each MU generated at the caller's terminal is output $\Delta \mathrm{ms}$ late at the terminal under the conventional control, the group synchronization quality between the caller and receiver is not good. To solve the problem, we need to delay the output timing of the MU by $2 \Delta$ ms. At each receiver's terminal, the receiver sees the caller show rock, paper or scissors on hearing "Rock, paper, scissors, go!" However, since the output timing of MUs generated at the receiver's terminal is delayed by $\Delta \mathrm{ms}$ at the terminal under the conventional control, the group synchronization quality between the caller and receiver is damaged. To improve the quality, we need to output the MUs on being captured. Thus, we propose group synchronization control considering difference of conversation roles.

\section{GROUP SYNCHRONIZATION CONTROL CONSIDERING DIFFERENCE OF CONVERSATION ROLES}

In the group synchronization control considering difference of conversation roles, the behavior of the control is different from terminal to terminal. At the caller's terminal, the output timing of each MU generated at the terminal is delayed by $2 \Delta \mathrm{ms}$ from the time at which the MU has been generated. MUs generated at each receiver's terminal are output at the terminal on being captured. At each terminal, MUs received from the other terminals are output in the same way as that under the conventional group synchronization control.

\section{EXPERIMENTAL SYSTEM AND SUBJECTIVE ASSESSMENT METHOD}

As shown in Fig. 2, our experimental system consists of three terminals (terminals 1, 2, and 3) and a network emulator (NIST Net [14]). Terminal 1 (CPU: Pentium4 processor at $2.4 \mathrm{GHz}$, RAM: 512 Mbytes) and terminals 2 and 3 (CPU: Pentium4 processor at $2.8 \mathrm{GHz}, \mathrm{RAM}$ : 512 Mbytes) are connected to each other via the network emulator by using 100BASE-T cables. NIST Net is employed to generate an additional delay for each MU transmitted among the three terminals according to the Pareto-normal distribution [14]. Each terminal has a headset with a microphone and a video camera. The terminal captures voice samples (PCM (Pulse Code Modulation), the average bit rate: $64 \mathrm{kbps}$ ) every $20 \mathrm{~ms}$, and it transmits the samples to the other terminals as a voice MU. Moreover, it inputs video pictures (MPEG-1 [15], GOP (Group Of Pictures): Intra-coded (I-) frames only ${ }^{1}$, the average bit rate: $922 \mathrm{kbps}$ ) as a video MU every $33 \mathrm{~ms}$. It should be noted that voice samples captured at each terminal are not output at the terminal, and the voice samples are output at only the other terminals. However, video pictures captured at each terminal are output at the terminal as well as the other terminals. Each MU includes a timestamp, which denotes the generation time of the MU. The MU is transferred by UDP. In the experiment, the averages of additional delays 1 and 2 in Fig. 2 are changed from $50 \mathrm{~ms}$ to $250 \mathrm{~ms}$ at intervals of $50 \mathrm{~ms}$ [12]. Furthermore, the standard deviations of additional

${ }^{1}$ In order to maintain the interactivity high, we deal with only Intra-coded (I-) frames.

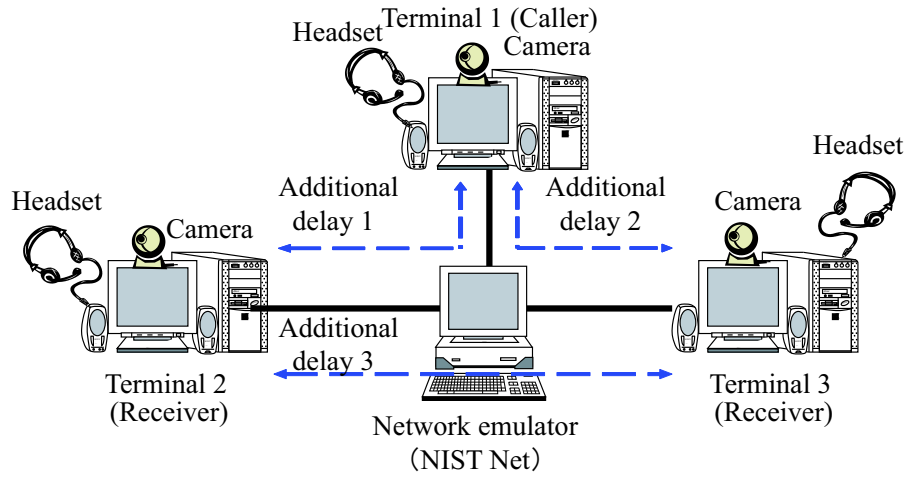

Fig. 2. Configuration of experimental System.

TABLE I

FIVE-GRADE IMPAIRMENT SCALE FOR DETERIORATION OWING TO NETWORK LATENCY.

\begin{tabular}{c|c}
\hline \hline Score & Description \\
\hline \hline 5 & Imperceptible \\
\hline 4 & Perceptible, but not annoying \\
\hline 3 & Slightly annoying \\
\hline 2 & Annoying \\
\hline 1 & Very annoying \\
\hline
\end{tabular}

delays 1 and 2 are set to $10 \mathrm{~ms}$, and additional delay 3 is set to $0 \mathrm{~ms}$.

In subjective assessment, we deal with three cases. In one case, group synchronization control is not exerted. In another case, the conventional group synchronization control is performed. In the other case, the group synchronization control considering difference of conversation roles is carried out. For the assessment, we have enhanced the single-stimulus method of ITU-R BT.500-11 [16]. In the method, each subject makes a conversation for 10 seconds on the condition that there is no additional delay, and then the subject does for the same duration by generating additional delays. He/she gives a score based on the five-grade impairment scale in Table I in terms of the group synchronization quality, interactivity, and comprehensive quality. The comprehensive quality is a synthesis of the group synchronization quality and interactivity. At first, each subject acts as the caller at terminal 1 or one of the receivers at terminal 2. Next, they swap their roles with each other and assess the group synchronization quality, interactivity, and comprehensive quality. The other receiver at terminal 3 is served by one of the authors. Subjects are twenty persons (men and women) whose ages are between 21 and 24 .

\section{ASSESSMENT RESULTS}

Here we present assessment results of the caller and then those of the receiver.

\section{A. Caller}

We show the Mean Opinion Scores (MOS [16]) of group synchronization quality, interactivity, and comprehensive quality of caller as a function of the average of additional delay 2 minus the average of additional delay 1 (called the difference in network latency here) in Figs. 3, 4, and 5, respectively. In the figures, we display the $95 \%$ confidence intervals (we also plot the $95 \%$ confidence intervals in the following figures). Furthermore, the case in which group synchronization control is not exerted is denoted by No control in this paper, the 
case in which the conventional group synchronization control is performed is denoted by Conventional control, and the case in which the group synchronization control considering difference of conversation roles is carried out is denoted by Proposed control.

From Fig. 3, we find that the MOS values of Proposed control are larger than those of Conventional control and No control. The reason is as follows. In the case of No control, the caller sees each receiver show rock, paper or scissors the same time as the round-trip delay between the caller's and receiver's terminals after the caller has shown rock, paper or scissors at the caller's terminal. The caller's motion is output $\Delta$ ms late at the caller's terminal under the conventional control. Thus, the difference between the time at which the caller shows rock, paper or scissors and the time at which the receivers show rock, paper or scissors under Conventional control is shorter than that under No control. And Proposed control has the smallest difference among three types of control since the control delays the output timing of each MU generated at the caller's terminal by $2 \Delta \mathrm{ms}$, which corresponds to the roundtrip delay, at the terminal. Thus, the MOS values of Proposed control are larger than those of Conventional control and No control.

We also observe in Fig. 3 that the MOS values of the three types of control become smaller as the difference in network latency becomes larger when the difference in network latency is positive. Furthermore, the MOS values hardly depend on the difference in network latency when the difference in network latency is negative. The reason is as follows. We instructed the subjects to pay attention to the difference between the time at which the caller or a receiver had shown rock, paper or scissors first and the time at which another receiver or the caller had shown rock, paper or scissors last in order to assess the group synchronization quality. Therefore, the MOS values depend on the larger delay between additional delays 1 and 2 . Note that additional delay 1 is larger than additional delay 2 when the difference in network latency is negative. Thus, the MOS values are almost constant and depend on only additional delay 1.

In Fig. 4, we see that there is no large difference in the MOS value of interactivity among the three types of control. The MOS values of the three types of control become smaller as the difference in network latency becomes larger when the difference in network latency is positive as in Fig. 3.

In Fig. 5, the MOS values of comprehensive quality have a similar tendency to those of group synchronization quality (see Fig. 3). That is, we see that Proposed control can maintain the QoE of caller higher than Conventional control and No control.

\section{B. Receiver}

We show the MOS values of group synchronization quality, interactivity, and comprehensive quality of receiver versus the difference in network latency in Figs. 6, 7, and 8, respectively.

Figure 6 reveals that the MOS values of group synchronization quality of Proposed control are higher than those of Conventional control. We also find that the MOS values of No control tend to be the highest among the three types of control when the average of additional delay 1 is $150 \mathrm{~ms}$ and $250 \mathrm{~ms}$. However, the difference in the MOS value between Proposed control and No control tends to be smaller than that of the caller, and the MOS values of Proposed control are larger than about 3 .

From Figs. 7 and 8, we see that the MOS values of interactivity and comprehensive quality are almost the same as those of group synchronization quality (see Fig. 6).

Moreover, we notice that the MOS values of comprehensive quality of receiver are larger than those of caller (see Figs. 5 and 8). The reason is as follows. At the caller's terminal, it takes about $2 \Delta$ ms to be output on the caller's screen from the moment the caller has shown rock, paper or scissors. However, each receiver's screen projects instantly the receiver's motion (i. e., showing rock, paper or scissors). That is, the interactivity of the receiver is largely different from that of the caller (see Figs. 4 and 7). Thus, the MOS values of comprehensive quality of receiver are larger than those of caller.

\section{CONCLUSIONS}

This paper proposed group synchronization control considering difference of conversation roles and examined the effects of the control by subjective assessment for networked rock-paper-scissors with voice and video. Assessment results showed that the proposed group synchronization control maintains the comprehensive quality higher than the conventional group synchronization control.

As the next step of our research, we plan to enhance the proposed group synchronization control by switching the three types of control (i.e., Proposed control, Conventional control, and No control) dynamically according to work contents.

\section{REFERENCES}

[1] F. Gong, "Multipoint audio and video control for packet-based multimedia conferencing," in Proceedings of ACM Multimedia'96, pp. 308-314, April 1996.

[2] H. Nakanishi, C. Yoshida, T. Nishimura, and T. Ishida, "FreeWalk: Supporting casual meetings in a network," in Proceedings of IEEE CSCW'96, pp. 308-314, April 1996.

[3] T. Kurita, "Effects of conversation roles on quality of multiparty audiovisual communication services," (in Japanese), IEICE Technical Report, CQ2005-32, July 2005.

[4] S. R. Ahuja and J. R. Ensor, "Coordination and control of multimedia conferencing," IEEE Communication Magazine, vol. 30, no. 5, pp. 3843, May 1992.

[5] Y. Ishibashi, M. Nagasaka, and N. Fujiyoshi, "Subjective assessment of fairness among users in multipoint communications," in Proceedings of ACM SIGCHI ACE'06, June 2006.

[6] R. Ohno, H. Aida, and T. Saito, "A media synchronization mechanism for a distributed multimedia system with interactive control," IEICE Transactions on Communications, vol. E78-B, no. 7, pp. 980-986, July 1995.

[7] I. F. Akyildiz and W. Yen, "Multimedia group synchronization protocols for integrated services networks," IEEE Journal on Selected Areas in Communications, vol. 14, no. 1, pp. 162-173, January 1996.

[8] Y. Ishibashi, A. Tsuji, and S. Tasaka, "A group synchronization mechanism for stored media in multicast communications," in Proceedings of INFOCOM'97, pp. 693-701, April 1997.

[9] Y. Ishibashi and S. Tasaka, "A group synchronization mechanism for live media in multicast communications," in Conference Record of IEEE GLOBECOM'97, pp. 746-752, November 1997.

[10] Y. Ishibashi and S. Tasaka, "A distributed control scheme for group synchronization in multicast communications," in Proceedings of ISCOM'97, pp. 313-323, November 1999.

[11] Y. Ishibashi, S. Tasaka, and Y. Tachibana, "Media synchronization and causality control for distributed multimedia applications," IEICE Transactions on Communications, vol. E84-B, no. 3, pp. 667-677, March 2001.

[12] K. Hosoya, Y. Ishibashi, S. Sugawara, and K. E. Psannis, "Effects of group synchronization control in networked virtual environments with avatars," in Proceedings of IEEE DS-RT'08, pp. 119-127, October 2008.

[13] Y. Ishibashi, S. Tasaka, and Y. Tachibana, "Adaptive causality and media synchronization control for networked multimedia applications," in Conference Record of IEEE ICC'01, pp. 952-958, June 2001.

[14] M. Carson and D. Santay, "NIST Net - A Linux-based network emulation tool," ACM SIGCOMM Computer Communication Review, vol. 33, no. 3, pp. 111-126, July 2003.

[15] ISO/IEC 11172, "Coding of moving pictures and associated audio for digital storage media at up to about $1.5 \mathrm{Mbit} / \mathrm{s}$ - Part 2: Video," Joint Technical Committee 1 for Information Technology, November 1993.

[16] ITU-R BT.500-11, "Methodoly for the subjective assessment of the quality of television picture," International Telecommunication Union, June 2002. 


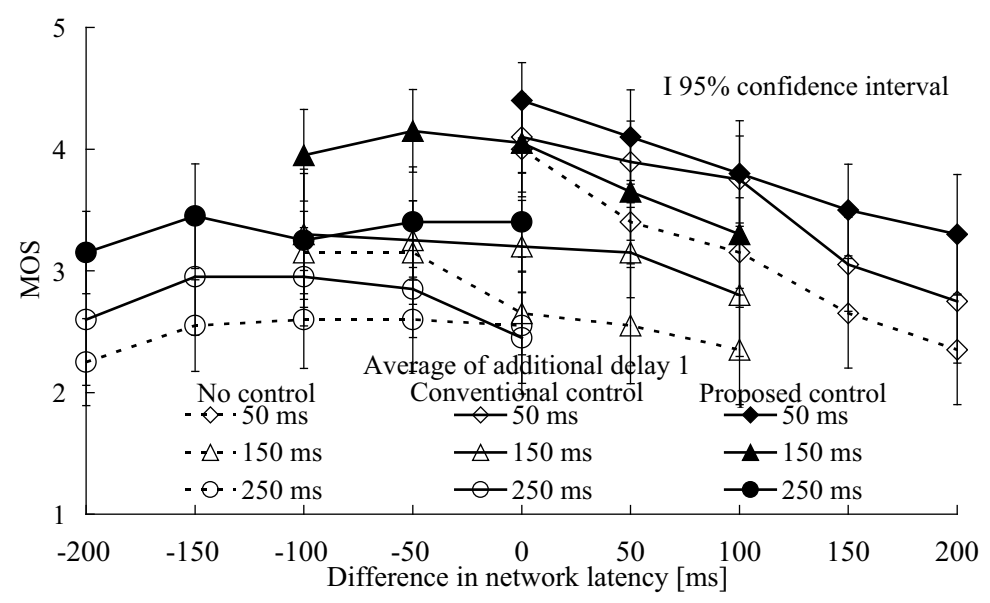

Fig. 3. MOS of group synchronization quality of caller versus difference in network latency.

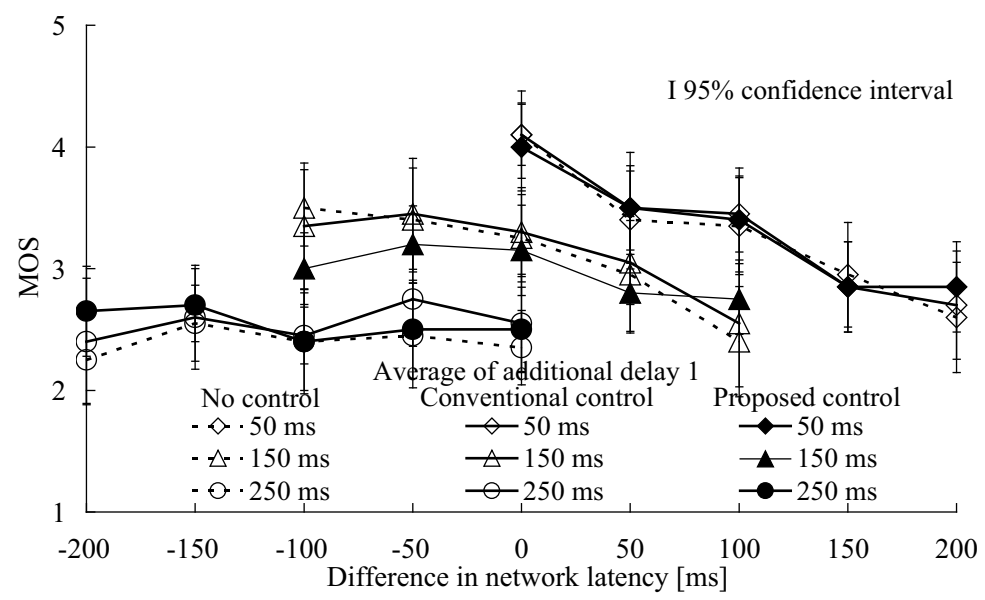

Fig. 4. MOS of interactivity of caller versus difference in network latency.

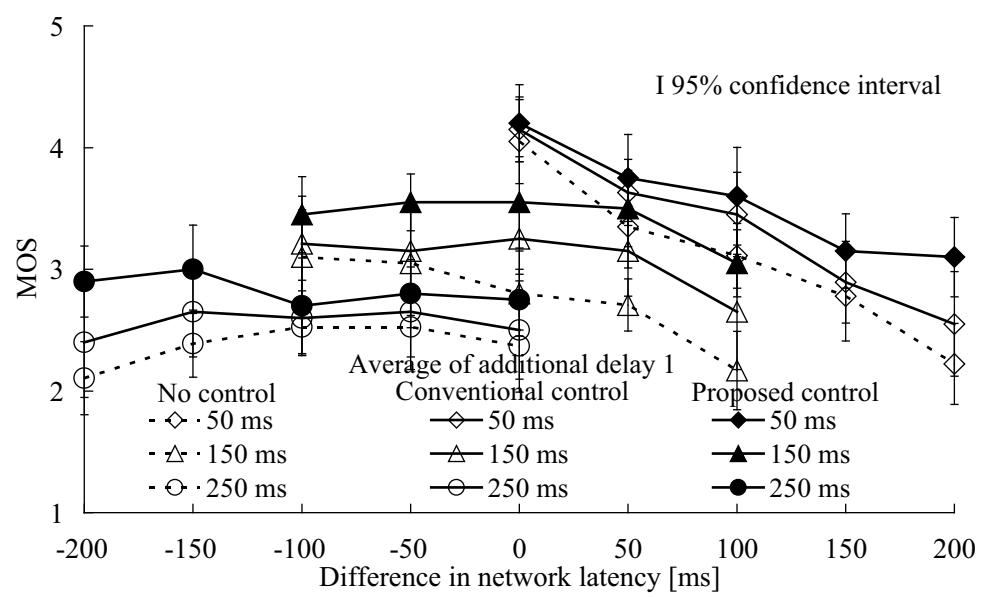

Fig. 5. MOS of comprehensive quality of caller versus difference in network latency. 


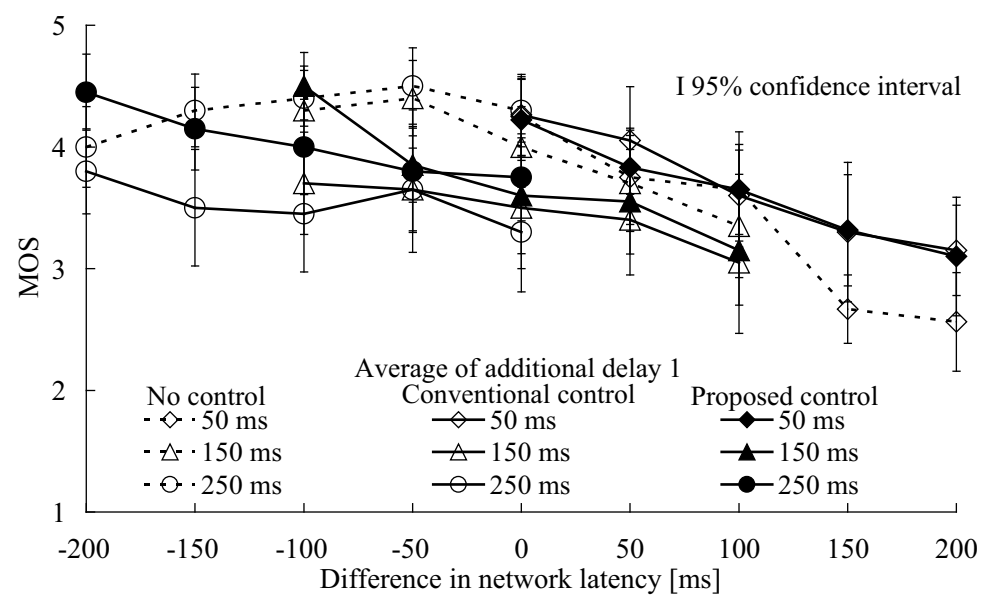

Fig. 6. MOS of group synchronization quality of receiver versus difference in network latency.

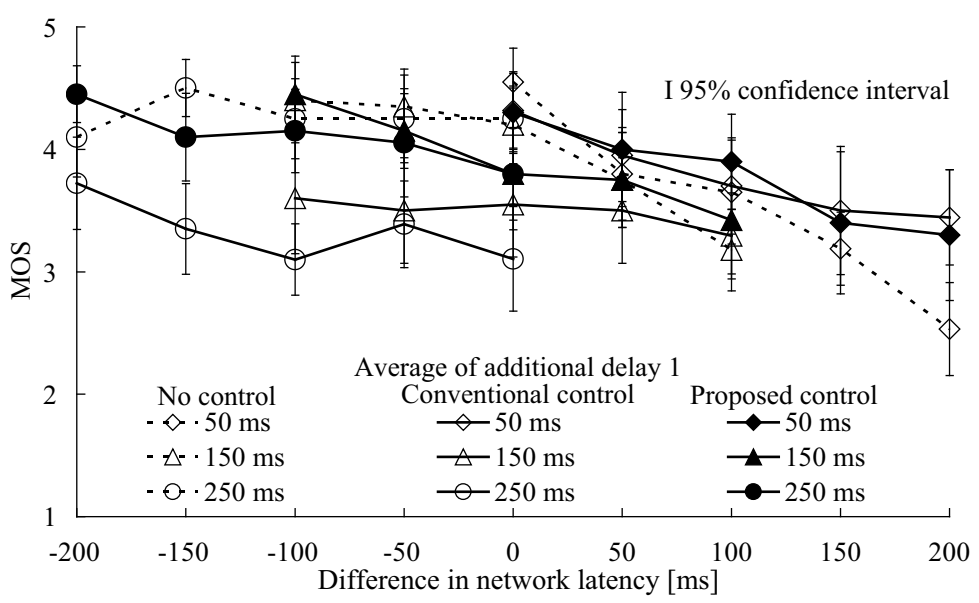

Fig. 7. MOS of interactivity of receiver versus difference in network latency.

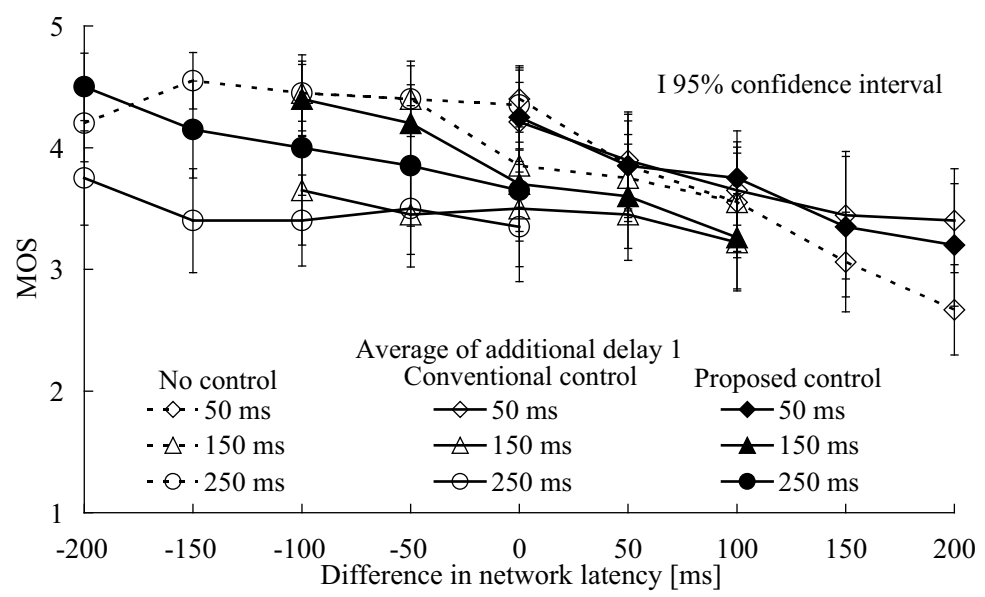

Fig. 8. MOS of comprehensive quality of receiver versus difference in network latency. 